\title{
NOTA PRELIMINAR SOBRE LOS DERMATOGLIFOS DIGITALES EN DOS POBLACIONES MAYAS DE YUCATÁN
}

Por Carlos SERRANo S.

Centro de Estudios Mayas, UNAM

Los datos que se exponen en esta nota constituyen el resultado preliminar de un estudio que se halla en elaboración sobre las características dermopapilares de los mayas de Yucatán.

Este trabajo ha sido realizado en el Centro de Estudios Mayas de la UNAM, en el marco de un plan de investigación más amplio que intenta, a través del estudio de los dermatoglifos, examinar el problema de las relaciones biológicas entre los distintos pueblos pertenecientes a la familia lingüística maya.

El análisis dermatoglífico parece especialmente adecuado para el discernimiento de distancias biológicas entre poblaciones humanas emparentadas ya que posee ciertas ventajas para penetrar en el tiempo y/o el espacio. Estas ventajas pueden resumirse como sigue (Newman, 1970, p. 167): 1) son rasgos poligénicos, y como tales, menos susceptibles de alteraciones a través de la deriva genética; 2) no sufren alteraciones posnatales, en contraste con los caracteres antropométricos; 3) parecen tener valores selectivos mínimos, y por lo tanto son relativamente no-adaptativos, y 4 ) las técnicas de formulación de los patrones dermatoglíficos poseen una objetividad bastante elevada que disminuye el error personal de observación. Estas ventajas, añade Newman (1970, p. 170), "recomiendan grandemente la aplicación del análisis dermatoglífico a los problemas de la historia racial en los indígenas de América Media".

$\mathrm{El}$ interés por los dermatoglifos en poblaciones del área maya data de principios del siglo (Wilder, 1904). Posteriormente se pueden mencionar los trabajos de Leche (1933, 1936), Cummins (1932), Cummins y Steggerda (1936) y Leche y colaboradores (1936), que examinaron sólo pequeñas series de individuos o se limitaron a un solo sexo; en algunos casos, los datos de ambos sexos se presentan 
conjuntamente, sin separar, lo cual pasa por alto que existen diferentes incidencias de los patrones digitales de acuerdo al sexo. Por otra parte, el análisis cuantitativo de los datos dermatoglíficos apenas ha sido tocado (Kalmus y colaboradores, 1964). Podemos considerar, por lo tanto, que la información sobre este carácter antropológico en el área maya es escasa e incompleta. Solamente se han reportado en detalle datos dermatoglíficos para algunos grupos mayas guatemaltecos (Newman, 1960).

A pesar de esta situación, el investigador antes citado ha intentado un análisis de poblaciones a partir de los dermatoglifos, para diferenciar regionalmente los distintos grupos de población en el área maya. Este enfoque, y la metodología empleada por aquel autor para el examen de los caracteres dermopapilares, ha puesto de relieve la utilidad de estos últimos para estudiar las afinidades bioantropológicas entre grupos humanos.

Esto no significa, por supuesto, desconocer el valor de la información que pueda obtenerse en otro tipo de estudios (serológicos, antropométricos, etcétera). En cuanto a los dermatoglifos, el trabajo antes citado muestra la importancia de obtener materiales más precisos y abundantes, orientados al estudio de los problemas de microevolución y diferenciación regional de poblaciones de la zona maya, objeto de nuestro interés.

\section{Material y métodos}

La obtención de las impresiones dígito-palmares se llevó al cabo a fines de 1971 en varias localidades del estado de Yucatán. La distribución por localidad se consigna en la tabla I.

TABLA I

PROCEDENCIA DE LOS SUJETOS

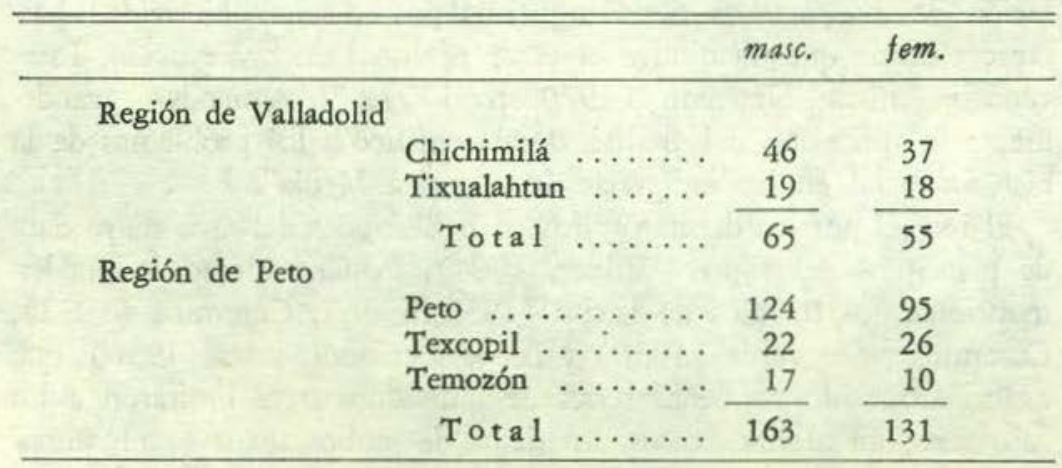


Las impresiones dermopapilares se obtuvieron en la población infantil de las escuelas rurales de las localidades visitadas. En su gran mayoría, los sujetos son oriundos del sitio donde fueron estudiados, y algunos de poblaciones circundantes de la misma región. Se incluyeron varios casos de individuos emparentados (hermanos del mismo o de distinto sexo). Los escolares fluctuaban en su inmensa mayoría entre los ocho y los doce años.

Según Lasker (1954, p. 358), en América Media contemporánea parece usual que el sitio de población, sea rancho, pueblo o cabecera, es coextensivo con la comunidad de reproducción (breeding community). En nuestro caso, aunque los individuos examinados integran la población infantil, podemos considerarlos como pertenecientes a comunidades de enlace matrimonial y por lo tanto, representantes de las poblaciones de donde proceden.

Para la tabulación de los datos obtenidos se han reunido, sin embargo, las series parciales de los sitios visitados, de acuerdo a la región, teniendo en cuenta la proximidad geográfica de su ubicación entre ellos.

Aunque el patrón de enlace matrimonial entre los mayas es en general endogámico, en las localidades visitadas la apertura de vías de comunicación ha roto el relativo aislamiento en que se habían mantenido, favoreciendo el intercambio genético entre diversas comunidades. Se puede considerar, por el mismo motivo, que el mestizaje biológico se ha incrementado. En efecto, a pesar de que todos los individuos examinados hablaban la lengua maya, algunos presentaban ciertas características somáticas indicadoras de mestizaje europeo.

Las impresiones dermopapilares se obtuvieron utilizando la técnica bien conocida que consiste en aplicar tinta sobre la palma y la superficie anterior de los dedos de la mano, y recoger la impresión sobre papel. Se hizo uso de un rodillo giratorio, descrito en otra parte (Serrano, 1971), con el cual se logran impresiones dígito-palmares de buena calidad.

El método utilizado para la identificación y clasificación de los diseños digitales es el descrito en la obra de Cummins y Midlo (1961); en esta ocasión sólo reportamos las principales categorías de patrones digitales (torbellinos, presillas cubitales y radiales, y arcos), cuya formulación es bastante consistente entre diferentes investigadores (figura 1 ).

Los datos que se exponen se refieren así, a las frecuencias de los dermatoglifos digitales y su examen a través de los índices de Cummins (pattern intensity), Furuhata y Dankmeijer, que expresan la incidencia relativa de los distintos tipos de diseños digitales. 

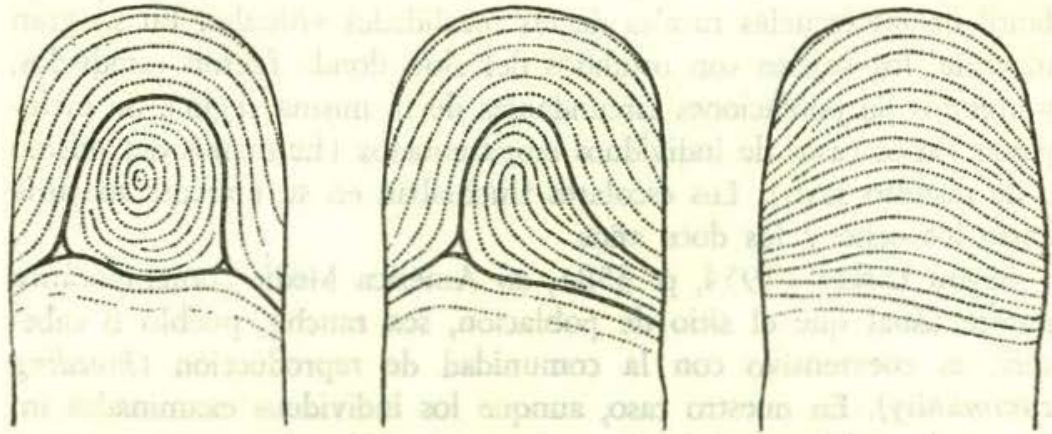

Figura 1. Los tres tipos básicos de dermatoglifos digitales: Torbellino, Presilla, Arco. (Según Cummins y Midlo, 1961, p. 56.)

El análisis de los dermatoglifos palmares y el estudio cuantitativo de las impresiones dígito-palmares serán objeto de un trabajo posterior.

\section{Resultados}

La frecuencia de los dermatoglifos digitales en las poblaciones estudiadas se consigna en la tabla II. Las cifras se refieren a las series separadas por sexo, así como de ambas reunidas, para facilitar la comparación con los datos publicados.

TABLA II

FRECUENCIA DE LOS DERMATOGLIFOS DIGITALES EN DOS POBLACIONES MAYAS DE YUCATÁN. \%

\begin{tabular}{|c|c|c|c|c|c|c|c|}
\hline \multirow[b]{2}{*}{ Población } & \multirow{2}{*}{ Sexo } & \multirow[b]{2}{*}{$n *$} & \multirow[b]{2}{*}{ Arcos } & \multicolumn{2}{|c|}{ Presillas } & \multirow[b]{2}{*}{ Total } & \multirow[b]{2}{*}{ Torbellinos } \\
\hline & & & & Cubitales & Radiales & & \\
\hline \multirow{3}{*}{ Chichimilá } & Masc. & 620 & 5.64 & 48.39 & 1.29 & 49.67 & 44.67 \\
\hline & Fem. & 550 & 4.18 & 56.73 & 2.54 & 59.27 & 36.55 \\
\hline & M. + F. & 1,170 & 4.96 & 52.31 & 1.88 & 54.19 & 40.85 \\
\hline \multirow{3}{*}{ Peto } & Masc. & 1,600 & 4.94 & 52.25 & 2.50 & 54.75 & 40.31 \\
\hline & Fem. & 1,260 & 5.00 & 60.08 & 1.82 & 61.90 & 33.10 \\
\hline & M. + F. & 2,860 & 4.97 & 55.70 & 2.20 & 57.90 & 37.13 \\
\hline
\end{tabular}

- Número total de dígitos. 
En la tabla III se anotan los principales índices de patrones digitales que permiten valorar mejor las diferencias entre nuestras series y su comparación con otros datos conocidos.

TABLA III

INDICES DE LOS PATRONES DIGITALES EN DOS POBLACIONES MAYAS DE YUCATÁN

\begin{tabular}{llccc}
\hline \hline Población & Sexo & $\begin{array}{c}I \\
\text { Cummins }\end{array}$ & $\begin{array}{c}I \\
\text { Furubata }\end{array}$ & $\begin{array}{c}I \\
\text { Dankmeijer }\end{array}$ \\
\hline \multirow{4}{*}{ Chichimilá } & Masc. & 13.90 & 89.93 & 12.63 \\
& Fem. & 13.24 & 61.67 & 11.44 \\
& M. + F. & 13.59 & 75.38 & 12.14 \\
\multirow{2}{*}{ Peto } & Masc. & 13.54 & 73.63 & 12.26 \\
& Fem. & 12.81 & 53.47 & 15.11 \\
& M. + F. & 13.22 & 64.13 & 13.39 \\
\hline
\end{tabular}

En las tablas IV y V se muestra la distribución porcentual de los dermatoglifos digitales en los diferentes dedos, para cada una de las muestras regionales. Se observará que los torbellinos fueron más frecuentes en los dedos IV y I en las series de Chichimilá, y en los mismos dígitos, pero a la inversa, en las de Peto. En todos los casos, las presillas fueron más comunes en los dígitos V y III, las presillas radiales casi exclusivas del dígito II y predominando los arcos en los dedos II y III.

En relación al lado, los torbellinos fueron más frecuentes, en general, en la mano derecha, para el sexo masculino, y a la inversa en el femenino. Las presillas, por el contrario, presentaron porcentajes mayores en la mano izquierda en las series masculinas, y en la mano derecha entre las femeninas. Estos resultados no son muy diferentes de los reportados para otras poblaciones.

$\mathrm{Si}$ consideramos las frecuencias de los diseños digitales en las series estudiadas, se notará que en todos los casos las presillas son el patrón predominante, acentuándose las cifras en el sexo femenino, conforme a la tendencia propia de este sexo en diversas poblaciones humanas. Aunque el porcentaje de torbellinos en nuestras series es menor que el de las presillas, alcanza, sin embargo, una cifra elevada, que en el caso de las series masculinas sobrepasa el $40 \%$ de los patrones digitales. Los arcos, tal como se ha visto en otras series mayas estudiadas, presentan una incidencia relativamente elevada, alrededor del $5 \%$. 
TABLA IV

DISTRIBUCIÓN PORCENTUAL DE DERMATOGLIFOS DIGITALES CHICHIMILÁ, YUC.

\begin{tabular}{|c|c|c|c|c|c|c|c|c|c|c|c|}
\hline \multirow[b]{3}{*}{ Digito } & \multirow[b]{3}{*}{ Lado } & \multicolumn{5}{|c|}{ Hombres } & \multicolumn{5}{|c|}{ Mujeres } \\
\hline & & \multirow[b]{2}{*}{ Arcos } & \multicolumn{2}{|c|}{ Presillas } & \multirow[b]{2}{*}{ Total } & \multirow{2}{*}{ Torbellinos } & \multirow[b]{2}{*}{ Arcos } & \multicolumn{2}{|c|}{ Presillas } & \multirow[b]{2}{*}{ Total } & \multirow[b]{2}{*}{ Torbellinos } \\
\hline & & & Cubitales & Radiales & & & & Cubitales & Radiales & & \\
\hline \multirow{3}{*}{ I } & Der. & 3.23 & 30.65 & $\longrightarrow$ & 30.65 & 66.13 & 1.82 & 50.91 & $\longrightarrow$ & 50.91 & 47.27 \\
\hline & Izq. & 3.23 & 41.94 & 1.61 & 43.55 & 53.23 & 1.82 & 47.27 & 1.82 & 49.09 & 49.09 \\
\hline & D. $+\mathrm{I}$. & 3.23 & 36.29 & 0.81 & 37.10 & 59.68 & 1.82 & 49.09 & 0.91 & 50.00 & 48.18 \\
\hline \multirow{3}{*}{ II } & Der. & 16.13 & 35.48 & - & 35.48 & 48.39 & 10.91 & 34.55 & 10.91 & 45.45 & 43.64 \\
\hline & Izq. & 11.29 & 46.77 & 9.68 & 56.45 & 32.26 & 10.91 & 41.82 & 10.91 & 52.73 & 36.36 \\
\hline & D. $+\mathrm{I}$. & 13.71 & 41.13 & 4.84 & 45.97 & 40.32 & 10.91 & 38.18 & 10.91 & 49.09 & 40.00 \\
\hline \multirow{3}{*}{ III } & Der. & 6.45 & 61.29 & - & 61.29 & 32.26 & 1.82 & 81.82 & $\longrightarrow$ & 81.82 & 16.36 \\
\hline & Izq. & 6.45 & 59.68 & 1.61 & 61.29 & 32.26 & 7.27 & 69.09 & $\longrightarrow$ & 69.09 & 23.64 \\
\hline & D. + I. & 6.45 & 60.48 & 0.81 & 61.29 & 32.26 & 4.55 & 75.45 & $\longrightarrow$ & 75.45 & 20.00 \\
\hline \multirow{3}{*}{ IV } & Der. & - & 30.65 & $\longrightarrow$ & 30.65 & 69.35 & - & 43.64 & - & 43.64 & 56.36 \\
\hline & Izq. & 3.23 & 33.87 & $\longrightarrow$ & 33.87 & 62.90 & - & 36.36 & 1.82 & 38.18 & 61.82 \\
\hline & D. $+\mathrm{I}$. & 1.59 & 32.26 & $\square$ & 32.26 & 66.13 & - & 40.00 & 0.91 & 40.91 & 59.09 \\
\hline \multirow{3}{*}{$\mathbf{v}$} & Der. & 3.23 & 67.74 & - & 67.74 & 29.03 & 1.82 & 83.64 & $\longrightarrow$ & 83.64 & 14.55 \\
\hline & Izq. & 3.23 & 75.81 & $\longrightarrow$ & 75.81 & 20.97 & 5.45 & 78.18 & - & 78.18 & 16.36 \\
\hline & D. + I. & 3.23 & 71.77 & & 71.77 & 25.00 & 3.64 & 80.91 & & 80.91 & 15.45 \\
\hline \multirow{3}{*}{$\begin{array}{c}\text { Todos } \\
\text { los dedos }\end{array}$} & Der. & 5.81 & 45.16 & - & 45.16 & 49.03 & 3.27 & 58.91 & 2.18 & 61.09 & 35.64 \\
\hline & Izq. & 5.48 & 51.61 & 2.58 & 54.19 & 40.32 & 5.09 & 54.54 & 2.91 & 57.45 & 37.45 \\
\hline & D. + I. & 5.64 & 48.39 & 1.29 & 49.67 & 44.67 & 4.18 & 56.73 & 2.54 & 59.27 & 36.55 \\
\hline
\end{tabular}


TABLA V

DISTRIBUCIÓN PORCENTUAL DE DERMATOGLIFOS DIGITALES PETO, YUC.

\begin{tabular}{|c|c|c|c|c|c|c|c|c|c|c|c|}
\hline \multirow[b]{3}{*}{ Digito } & \multirow[b]{3}{*}{ Lado } & \multicolumn{5}{|c|}{ Hombres } & \multicolumn{5}{|c|}{ Mujeres } \\
\hline & & \multirow[b]{2}{*}{ Arcos } & \multicolumn{2}{|c|}{ Presillas } & \multirow[b]{2}{*}{ Total } & \multirow[b]{2}{*}{ Torbellinos } & \multirow[b]{2}{*}{ Arcos } & \multicolumn{2}{|c|}{ Presillas } & \multirow[b]{2}{*}{ Total } & \multirow[b]{2}{*}{ Torbellinos } \\
\hline & & & Cubitales & Radiales & & & & Cubitales & Radiales & & \\
\hline \multirow{3}{*}{ I } & Der. & 1.25 & 26.87 & 0.62 & 27.50 & 71.25 & 4.76 & 38.10 & 1.59 & 39.68 & 55.55 \\
\hline & Izq. & 3.12 & 37.50 & 1.25 & 38.75 & 58.13 & 6.35 & 34.13 & 0.79 & 34.92 & 58.73 \\
\hline & D. $+\mathrm{I}$. & 2.19 & 32.19 & 0.94 & 33.12 & 64.69 & 5.56 & 36.11 & 1.19 & 37.30 & 57.14 \\
\hline \multirow{3}{*}{ II } & Der. & 16.25 & 30.62 & 10.00 & 40.62 & 43.12 & 14.28 & 50.00 & 2.38 & 52.38 & 33.33 \\
\hline & Izq. & 18.12 & 35.00 & 10.00 & 45.00 & 36.87 & 15.08 & 42.06 & 9.52 & 51.59 & 33.33 \\
\hline & D. + I. & 17.19 & 32.81 & 10.00 & 42.81 & 40.00 & 14.68 & 46.03 & 5.95 & 51.98 & 33.33 \\
\hline \multirow{3}{*}{ III } & Der. & 3.75 & 74.38 & - & 74.38 & 21.87 & 2.38 & 85.71 & $\longrightarrow$ & 85.71 & 11.90 \\
\hline & Izq. & 3.12 & 68.75 & 0.62 & 69.38 & 27.50 & 3.97 & 73.02 & 0.79 & 73.81 & 22.22 \\
\hline & D. + I. & 3.44 & 71.56 & 0.31 & 71.87 & 24.69 & 3.17 & 79.36 & 0.40 & 79.76 & 17.06 \\
\hline \multirow{3}{*}{ IV } & Der. & 1.25 & 41.25 & 1.25 & 42.50 & 56.25 & 0.79 & 50.00 & 1.59 & 51.59 & 47.62 \\
\hline & Izq. & - & 47.50 & & 47.50 & 52.50 & 1.59 & 50.79 & 1.59 & 52.38 & 46.03 \\
\hline & D. + I. & 0.62 & 44.37 & 0.62 & 45.00 & 54.37 & 1.19 & 50.40 & 1.59 & 51.98 & 46.83 \\
\hline \multirow{3}{*}{ V } & Der. & 1.25 & 75.63 & 1.25 & 76.88 & 21.87 & - & 89.68 & - & 89.68 & 10.32 \\
\hline & Izq. & 1.25 & 85.00 & $\longrightarrow$ & 85.00 & 13.75 & 0.79 & 87.30 & - & 87.30 & 11.90 \\
\hline & D. + I. & 1.25 & 80.31 & 0.62 & 80.94 & 17.81 & 0.40 & 88.49 & $\longrightarrow$ & 88.49 & 11.11 \\
\hline Todos & Der. & 4.75 & 49.75 & 2.62 & 52.37 & 42.87 & 4.44 & 62.70 & 1.11 & 63.81 & 31.74 \\
\hline \multirow[t]{2}{*}{ los dedos } & Izq. & 5.12 & 54.75 & 2.37 & 57.12 & 37.75 & 5.56 & 57.46 & 2.54 & 60.00 & 34.44 \\
\hline & D. + I. & 4.94 & 52.25 & 2.50 & 54.75 & 40.31 & 5.00 & 60.08 & 1.82 & 61.90 & 33.10 \\
\hline
\end{tabular}


Los porcentajes elevados de torbellinos caracterizan en su conjunto a las poblaciones de la zona maya, en particular a los pueblos de los Altos de Chiapas (tabla 5), que presentan las cifras más altas en las series mesoamericanas ( $>$ de $40 \%$ ). Entre los mayas de Yucatán y de Guatemala, el porcentaje de torbellinos disminuye, aunque se mantiene alrededor de la cifra anotada. Para poblaciones no-mayas mesoamericanas, el rango de variación de los torbellinos, con raras excepciones, es de $28-40 \%$. Si se considera el sexo femenino exclusivamente, estas cifras deben reducirse como consecuencia de la tendencia antes mencionada en este sexo de presentar porcentajes menores de este diseño digital.

$\mathrm{Si}$ atendemos al índice de Furuhata, ${ }^{1}$ que enfatiza la frecuencia relativa de torbellinos en una población, los valores obtenidos en nuestras series caen en el campo de variación que presentan para tal índice las poblaciones mayas. Para las series masculinas encontramos cifras que van de 70.42 (tzeltales) a 120.75 (mayas yucatecos), denotando las frecuencias relativamente elevadas de torbellinos. En series indígenas mesoamericanas no-mayas, la variación del mismo índice se establece, según los datos hasta ahora conocidos, entre 55.49 (tarascos) y 70.17 (mixtecos).

Podemos encontrar indicaciones semejantes a través del examen del índice de Cummins (pattern intensity index). ${ }^{2}$ Las variaciones en nuestras series se establecen en el sentido de valores más elevados para las series masculinas de ambas poblaciones estudiadas, lo cual traduce la incidencia mayor de torbellinos en este sexo. Por otra parte, si comparamos las series reunidas de ambos sexos de Chichimilá con las correspondientes de Peto, el índice de Cummins es más elevado en aquellas. La reducción del porcentaje de torbellinos, y por lo tanto del índice de Cummins, quizá podría reflejar los efectos de un mayor mestizaje europeo. En efecto, algunos caracteres somatoscópicos observados en la población de la región de Peto indican una mezcla mayor que en la región de Chichimilá. Este mismo hecho puede explicar quizá las diferencias que encontramos en el índice de Cummins en relación a otras series estudiadas antes.

En la tabla VII se han reunido los datos sobre el índice de Cummins en poblaciones mayas, de acuerdo a los datos publicados (Newman, 1970; Coope y Roberts, 1971), que nos permiten ubicar

$1 \mathrm{~T} / \mathrm{P}$, o sea, la relación porcentual de los torbellinos respecto a las presillas.

2 Este índice se basa en la secuencia de complejidad creciente de los diseños digitales, según el número de deltas o trirradios (puntos donde convergen tres crestas papilares). Los torbellinos están limitados por dos trirradios y las presillas por uno, los arcos no presentan por lo general ningún trirradio (figura 1). El índice se expresa entonces: $2 \%$ torbellinos $+1 \%$ presillas : 10 . 
TABLA VI

FRECUENCIA DE DERMATOGLIFOS DIGITALES EN POBLACIONES DEL ÁREA MAYA *

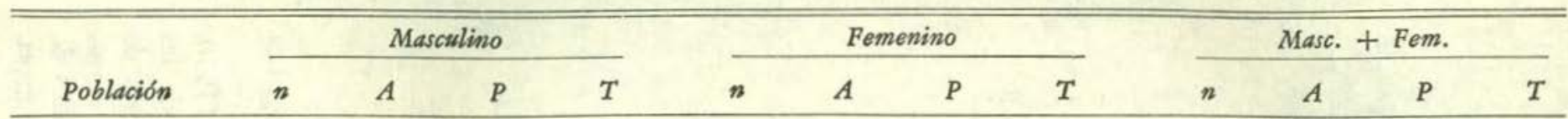

Tierras altas

septentrionales

$\begin{array}{lrlll}\text { Amatenango } & 49 & 1.2 & 46.4 & 52.4 \\ \text { Zinacantan } & 24 & 2.2 & 48.2 & 49.6 \\ \text { Hixtan } & 50 & 2.5 & 49.6 & 48.9 \\ \text { Chamula } & 100 & 3.4 & 52.8 & 43.8 \\ \text { Tezltales } & 47 & 2.8 & 57.0 & 40.2 \\ \text { Tzotziles } & 230 & 7.2 & 57.0 & 35.8 \\ \text { Tzeltales } & 62 & 5.0 & 52.6 & 42.4 \\ \text { Zinacantecos } & 90 & 2.8 & 46.8 & 50.4 \\ \text { Tzeltal-tzotzil } & 90 & 6.5 & 58.9 & 34.7 \\ \text { Soloma } & 90 & 2.4 & 48.8 & 48.7\end{array}$

52.4

49.6

40.2

35.8

42.4

$\begin{array}{llllllll}88 & 7.40 & 49.5 & 43.1 & 318 & 7.3 & 54.9 & 37.8\end{array}$

0.4

$\begin{array}{llllllll}21 & 2.9 & 39.4 & 57.8 & 111 & 2.8 & 45.4 & 51.8\end{array}$

Soloma

48.7

$\begin{array}{lllllll}2.0 & 54.9 & 43.1 & 112 & 2.3 & 50.0 & 47.6\end{array}$

Tierras altas

meridionales

$\begin{array}{lllll}\text { Santa Clara } & 68 & 5.6 & 54.9 & 39.5\end{array}$

$\begin{array}{lllll}\text { Solola } & 82 & 6.3 & 53.7 & 40.0\end{array}$

$\begin{array}{lllll}\text { Patzún } & 72 & 6.9 & 58.3 & 34.8\end{array}$

$\begin{array}{llllllll}32 & 14.0 & 49.9 & 36.1 & 104 & 9.1 & 55.7 & 35.2\end{array}$

Tierras bajas

de Yucatán

Hacienda Acu

Chichén Itzá

$\begin{array}{llll}25 & 6.4 & 42.4 & 51.2\end{array}$

Chichimilá +

$\begin{array}{lll}62 & 5.6 & 49.7\end{array}$

44.7

$\begin{array}{lll}160 & 4.9 & 54.8\end{array}$

40.3

$\begin{array}{ll}55 & 4.2\end{array}$

61.9

36.5
33.1

127

$\begin{array}{ll}7.6 & 59.2\end{array}$

33.2

Peto +

- Coope y Roberts, 1971 p. 411.

+ Presente estudio. 
TABLA VII

INDICE DE CUMMINS (PATTERN INTENSITY INDEX) EN POBLACIONES DEL ÁREA MAYA *

\begin{tabular}{|c|c|c|c|c|c|c|}
\hline Población & $n$ & masc. & $n$ & fem. & $n$ & $m .+f$. \\
\hline \multicolumn{7}{|l|}{$\begin{array}{l}\text { Tierras altas } \\
\text { septentrionales }\end{array}$} \\
\hline Amatenango & 49 & 15.4 & & & & \\
\hline Zinacantan & 24 & 14.7 & & & & \\
\hline Huixtan & 50 & 14.6 & & & & \\
\hline Chamula & 47 & 13.7 & & & & \\
\hline Tzeltal & 100 & 14.0 & & & & \\
\hline Tzotzil & 230 & 12.9 & 88 & 13.6 & 318 & 13.1 \\
\hline Tzeltal & 62 & 13.7 & & & & \\
\hline Zinacantecos & 90 & 14.8 & 21 & 15.5 & 111 & 14.9 \\
\hline Tzeltal-tzotzil & & & & & 23 & 14.2 \\
\hline Tzotzil-tzeltal & 90 & 13.5 & & & & \\
\hline Soloma & 90 & 14.5 & 24 & 14.2 & 114 & 14.4 \\
\hline Varios & 13 & 16.0 & & & & \\
\hline
\end{tabular}

Tierras altas

meridionales

\begin{tabular}{llllllll} 
Santa Clara & 68 & 13.2 & & & & \\
Solola & 82 & 13.1 & & & & \\
Patzún & 72 & 12.6 & 35 & 12.5 & 107 & 12.6 \\
Varios & 25 & 13.3 & & & & \\
\hline
\end{tabular}

Tierras bajas

de Yucatán

Hacienda Acu

Chichén Itzá

Chichimilá †

Peto +

$\begin{array}{rrrrrr}25 & 12.7 & & & & \\ 62 & 13.9 & 55 & 13.2 & 117 & 12.6 \\ 160 & 13.5 & 126 & 12.8 & 286 & 13.6\end{array}$

* Coope y Roberts, 1971, p. 414.

+ Presente estudio.

nuestros resultados en el contexto de la variabilidad dermatoglífica de la zona maya.

Se puede observar, como ha sido hecho notar por Newman (1970, p. 174) que los mayas de las tierras altas meridionales y de las tierras bajas de Yucatán constituyen un área con bajos valores de pattern intensity diferenciándose netamente de los grupos mayas de las tierras altas septentrionales. Estos últimos presentan índices de intensidad de patrón más elevados que otros pueblos de lengua maya. 
Nuestros resultados caben en general dentro de este esquema, aunque si sólo consideramos las series masculinas, los valores encontrados son superiores al límite $<13.40$ propuesto por el citado investigador para diferenciar las poblaciones mayas de Yucatán y Guatemala, de las que habitan en los Altos de Chiapas. Consideramos, sin embargo, que los datos recabados hasta ahora aún son insuficientes para precisar las diferencias regionales y, en su caso, los gradientes de este carácter en dicha área geográfica.

Por último, en lo que se refiere al índice de Dankmeijer, ${ }^{3}$ las series mayas estudiadas presentan cifras bastante elevadas, que expresan el porcentaje relativamente alto de arcos. Las frecuencias de arcos parecen ser especialmente útiles para diferenciar las poblaciones del área maya. Según Coope y Roberts (1971, p. 410), los mayas de las tierras altas septentrionales, con excepción de tzeltales y tzotziles, tienden a poseer frecuencias más bajas de arcos, y son así más similares a los no-mayas que a los pueblos de habla maya de las tierras altas del sur y de las tierras bajas de Yucatán, quienes tienen una frecuencia más elevada de arcos. Encontramos entonces la misma indicación de diferencias dermatoglíficas entre las poblaciones de los Altos de Chiapas y los grupos vecinos de lengua maya.

Hemos intentado en este breve trabajo situar nuestros datos en el campo de variación dermatoglífica de las poblaciones de la zona maya, en un nivel elemental de comparación de frecuencias de diseños digitales. Debe señalarse, sin embargo, la necesidad de efectuar estudios más profundos y obtener nuevos materiales de trabajo, dando por sentado que el análisis de los dermatoglifos es una útil herramienta para demostrar relaciones entre poblaciones, que en nuestro caso atañe especialmente a los grupos pertenecientes a la familia lingüística maya.

\section{BIBLIOGRAFIA}

\section{COOPE, E. Y D. F. ROBERTS}

1971 "Dermatoglyphics Studies of Populations in Latin America", en The ongoing evolution of Latin American Populations, ed. F. M. Salzano, pp. 405-53. Charles C Thomas, Publisher. Springfield.

CUMmins, $\mathrm{H}$.

1932 "Dermatoglyphics in Indians of Southern Mexico and Central America (Santa Eulalia, Tzeltal, Lacandon and Maya Tribes)", Middle American Research Institute, publ, núm. 4, pp. 181-208. New Orleans.

- A/T, o sea, la relación porcentual de los arcos respecto a los torbellinos. 
Cummins, H. y M. STEgGerda

1936 "Finger Prints in Maya Indians", Middle American Research Series, núm. 7, pp. 103-26. New Orleans.

Cummins, H. y CH. MidLo

1961 Finger prints, palms and soles. An introduction to dermatoglyphics. Dover Publications, Inc., New York.

Kalmus, H., A. L. dB Garay, U. Rodartb y L. COBO

1964 "The Frequency of PTC Tasting, Hard ear Wax, Colour Blindness and Other Genetical Characters in Urban and Rural Mexican Populations", Human Biology, vol. 36, pp. 134-145.

LASKER, G. W.

1954 "Human Evolution in Contemporary Communities", S.W. Journal of Anthropology, vol. 10, pp. 353-65.

LECHB, S. M.

1933 "Dermatoglyphics and Functional Lateral Dominance in Mexican Indians (Mayas and Tarahumaras)", Middle American Research Series, núm. 5, pp. 29-42. New Orleans.

1936 "Dermatoglyphics and Functional Lateral Dominance in Mexican Indians: IV. Chamulas. Anthropometry of the Chamulas", en: Measures of Men. Middle American Research Series, publ. núm. 7, pp. 287-312.

LeCHe, S. M., H. N. GOUld y D. TharP

1944 "Dermatoglyphics and Functional Lateral Dominances in Mexican Indians. V. Zinacantecs, Huixtecs, Amatenangos and Finca Tzeltals with the Anthropometry of these four Groups", Middle American Research Records, vol. 1, núm. 6, pp. 21-64.

NEWMAN, M. T.

1960 "Population Analysis of Finger and Palm Prints in Highland and Lowland Maya Indians", Amer. J. Phys. Atbrop., vol. 18, pp. 45-58.

1970 "Dermatoglyphics", en: Handbook of Middle American Indians, Physical Anthropology, vol. 9, pp. 167-79.

SERRANO, C.

1971 "Los Dermatoglifos Digitales en la Población Masculina de Cholula, Pue." Anales del Inst. Nal. de Antrop. e Hist., t. II, $7^{\text {a }}$ ep., pp. 59-66.

WILDER, H. H.

1904 "Racial Differences in Palm and Sole Configuration", Amer. Antbrop., vol. VI, pp. 244-93. 